\title{
SOME BESSEL FUNCTION INTEGRALS
}

\author{
by T. M. MACROBERT
}

(Received 16th June, 1955)

The basic formula to be proved is

$$
\int_{0}^{\infty} E\left(p ; \alpha_{r}: q ; \rho_{s}: z \operatorname{sech}^{2} u\right)(\sinh u)^{2 n-1} \cosh u d u=\frac{1}{2} \Gamma(n) z^{n} E\left(p ; \alpha_{r}-n: q ; \rho_{s}-n: z\right), \ldots(1)
$$

where $p \geqq q+1, z \neq 0,|\operatorname{amp} z|<\pi, R(n)>0, R\left(\alpha_{r}-n\right)>0, r=1,2, \ldots, p$. For other values of $p$ and $q$ the result holds if the integral converges. From this formula some results, involving Bessel functions and Confluent Hypergeometric functions, will be deduced.

In the formula

$$
\int_{0}^{\infty} e^{-\mu / z} \mu^{n-1} d \mu=\Gamma(n) z^{n}
$$

where $R(z)>0, R(n)>0$, replace $\mu$ by $\lambda-1$ and it can be written

$$
\int_{1}^{\infty} E(:: z / \lambda)(\lambda-1)^{n-1} d \lambda=\Gamma(n) z^{n} E(:: z)
$$

and, on generalising, this gives

$$
\int_{1}^{\infty} E\left(p ; \alpha_{r}: q ; \rho_{s}: z / \lambda\right)(\lambda-1)^{n-1} d \lambda=\Gamma(n) z^{n} E\left(p ; \alpha_{r}-n: q ; \rho_{s}-n: z\right),
$$

where $p \geqq q+1, z \neq 0,|\operatorname{amp} z|<\pi, R(n)>0, R\left(\alpha_{r}-n\right)>0, r=1,2, \ldots, p$. For other values of $p$ and $q$ the result holds if the integral converges. Formula (1) is obtained by putting $\lambda=\cosh ^{2} u$.

Note 1. In the process of increasing $q$ while $p$ remains fixed the formula used is

$$
\frac{1}{2 \pi i} \int e^{\zeta \zeta^{-\rho}} d \zeta=\frac{1}{\Gamma(\rho)}
$$

where the integral starts from $-\infty$ on the $\xi$-axis, passes round the origin in the positive direction, and returns to $-\infty$ on the $\xi$-axis, and amp $\zeta=0$ to the right of the origin. In deriving the case, $p=0, q=1$, from (2) $z$ should be taken to be real and positive and the contour should be replaced by a line parallel to and to the right of the imaginary axis. The integral (4) then converges if $R(\rho)>0$.

Note 2. If $\lambda$ is replaced by $1 / \lambda$ in (3), the resulting formula is a particular case of the formula given on page 118 of Volume I of these Proceedings, or of Ragab's more general formula (2) on page 77 of the present volume. By replacing the variables of integration in these formulae by $\operatorname{sech}^{2} u$, more general integrals of the same type as (1) can be obtained.

Now in (3) put $p=0, q=1, \rho_{1}=\frac{3}{2}$ and replace $n$ by $\frac{1}{2}-n, \lambda$ by $\xi^{2}$ and $z$ by $4 / x^{2}$; so obtain. ing the known formula*

$$
\int_{1}^{\infty} \frac{\sin (x \xi)}{\left(\xi^{2}-1\right)^{n+\frac{1}{1}}} d \xi=\frac{1}{2} \Gamma\left(\frac{1}{2}\right) \Gamma\left(\frac{1}{2}-n\right)\left(\frac{x}{2}\right)^{n} J_{n}(x)
$$

where $x$ is real and positive and $-\frac{1}{2}<R(n)<\frac{1}{2}$.

\footnotetext{
* Titchmarsh, E.C., Introduction to the Theory of Fourier Integrals, p. 200.
} 
In what follows the formulae

$$
\begin{array}{r}
\cos m \pi E\left(\frac{1}{2}+m, \frac{1}{2}-m:: 2 z\right)=\sqrt{ }(2 \pi z) e^{z} K_{m}(z), \ldots \ldots \ldots \ldots \\
E\left(\frac{1}{2}-k+m, \frac{1}{2}-k-m:: z\right)=\Gamma\left(\frac{1}{2}-k+m\right) \Gamma\left(\frac{1}{2}-k-m\right) z^{-k} e^{1 z} W_{k, m}(z)
\end{array}
$$

will be required.

In (1) put $p=2, q=0, \alpha_{1}=\frac{1}{2}+m, \alpha_{2}=\frac{1}{2}-m$, and replace $n$ by $k$ and $z$ by $2 z ;$ then, from (6) and (7),

$$
\begin{aligned}
\int_{0}^{\infty} e^{2 \operatorname{sech}^{2} u} & K_{m}\left(z \operatorname{sech}^{2} u\right)(\sinh u)^{2 k-1} d u \\
= & \frac{\cos m \pi}{\sqrt{(2 \pi z)}} \frac{1}{2} \Gamma(k) \Gamma(1-k+m) \Gamma\left(\frac{1}{2}-k-m\right) e^{z} W_{k, m}(2 z),
\end{aligned}
$$

where $z \neq 0,|\operatorname{anp} z|<\pi, R(k)>0, R(k \pm m)<!$

Again, in (1) put $p=2, q=0, \alpha_{1}=\frac{1}{2}+k+m, \alpha_{2}=1,+k-m$ and replace $z$ by $2 z$ and $n$ by $k$; then

$$
\int_{0}^{\infty} e^{z \operatorname{sech}^{2} u} W_{-k, m}\left(2 z \operatorname{sech}^{2} u\right)(\tanh u)^{2 k-1} d u=\frac{\Gamma(k) \sqrt{ }(2 \pi z) e^{z} K_{m}(z)}{2 \cos m \pi \Gamma\left(\frac{1}{2}+k+m\right) \Gamma\left(\frac{1}{2}+k-m\right)},
$$

where $z \neq 0,|\operatorname{amp} z|<\pi, R(k)>0, R\left(\frac{1}{2} \pm m\right)>0$.

Finally, let $p=0, q=1, \rho_{1}=m+1$, and replace $z$ by $4 / x^{2}$; then, if $x$ is real and positive,

$$
\int_{0}^{\infty} J_{m}(x \cosh u)(\sinh u)^{2 n-1}(\cosh u)^{1-m} d u=2^{n-1} \Gamma(n) x^{-n} J_{m-n}(x),
$$

provided that $R(n)>0, R(m-2 n)>-\frac{1}{2}$.

UNIVERSITY OF GLasgow 